\title{
Rate of convergence from the rotating Euler and shallow water equations to the rotating lake equations
}

Peng Cheng ${ }^{1,2}$, Jianwei Yang ${ }^{2^{*}}$ (D) and Dan Bai ${ }^{1}$

\section{"Correspondence:}

yangjianwei@ncwu.edu.cn

${ }^{2}$ College of Mathematics and

Information Science, North China

University of Water Resources and Electric Power, Zhengzhou, Henan

Province 450045, P.R. China

Full list of author information is

available at the end of the article

\begin{abstract}
The rate of convergence from the rotating shallow water and Euler equations to the rotating lake equations is obtained when the Froude number limit is considered.
\end{abstract}

MSC: 35B25; 35Q31; 76B15

Keywords: Euler and shallow water equations; lake equations; rate of convergence; zero Froude number limit

\section{Introduction and main results}

The inviscid rotating Euler and shallow water equations read, in a bounded twodimensional domain $\Omega$ :

$$
\begin{aligned}
& \partial_{t} h^{\epsilon}+\operatorname{div}\left(h^{\epsilon} u^{\epsilon}\right)=0, \\
& \partial_{t}\left(h^{\epsilon} u^{\epsilon}\right)+\operatorname{div}\left(h^{\epsilon} u^{\epsilon} \otimes u^{\epsilon}\right)+h^{\epsilon}\left(u^{\epsilon}\right)^{\perp}+h^{\epsilon} \frac{\nabla\left(\left(h^{\epsilon}\right)^{\gamma-1}-h_{0}^{\gamma-1}\right)}{\epsilon^{2}}=0,
\end{aligned}
$$

with initial and boundary conditions

$$
\left.\left(h^{\epsilon}, u^{\epsilon}\right)\right|_{t=0}=\left(h_{0}^{\epsilon}(x), u_{0}^{\epsilon}(x)\right),\left.\quad h^{\epsilon} u^{\epsilon} \cdot n\right|_{\partial \Omega}=0,
$$

where $\gamma \geq 2$. In (1.1)-(1.3), the unknowns $h^{\epsilon}=h^{\epsilon}(x, t)$ and $u^{\epsilon}=u^{\epsilon}(x, t)=\left(u_{1}^{\epsilon}(x, t), u_{2}^{\epsilon}(x, t)\right)$ denote the height of the water and the horizontal component of the fluid velocity, respectively. The orthogonal velocity is denoted by

$$
\left(u^{\epsilon}\right)^{\perp}=\left(\begin{array}{cc}
0 & -1 \\
1 & 0
\end{array}\right)\left(\begin{array}{l}
u_{1}^{\epsilon} \\
u_{2}^{\epsilon}
\end{array}\right)=\left(-u_{2}^{\epsilon}, u_{1}^{\epsilon}\right)
$$

and the strictly positive function $h_{0}=h_{0}(x)$ describes the bottom topography. The parameter $\epsilon>0$ is the Froude number measuring the inverse pressure forcing. Moreover, the energy for the system (1.1)-(1.3) can be defined as follows:

$$
E^{\epsilon}(t)=\int_{\Omega}\left(\frac{1}{2} h^{\epsilon}\left|u^{\epsilon}\right|^{2}+\frac{1}{\epsilon^{2}} \Phi\left(h^{\epsilon}\right)\right) d x,
$$

(c) The Author(s) 2017. This article is distributed under the terms of the Creative Commons Attribution 4.0 International License (http://creativecommons.org/licenses/by/4.0/), which permits unrestricted use, distribution, and reproduction in any medium, provided you give appropriate credit to the original author(s) and the source, provide a link to the Creative Commons license, and indicate if changes were made. 
where the potential energy

$$
\Phi\left(h^{\epsilon}\right)=\frac{1}{\gamma}\left(\left(h^{\epsilon}\right)^{\gamma}-h_{0}^{\gamma}-\gamma\left(h_{0}\right)^{\gamma-1}\left(h^{\epsilon}-h_{0}\right)\right)
$$

is a convex function with minimum occurring at $h^{\epsilon}=h_{0}$ which satisfies $\Phi\left(h^{\epsilon}\right) \geq 0$. It is easy to see that the energy inequality associated with system (1.1)-(1.3) reads

$$
\frac{d}{d t} E^{\epsilon}(t)=0
$$

By the initial energy bound (2.1) below and the following elementary convexity inequality:

$$
\frac{1}{\gamma}\left|h^{\epsilon}-h_{0}\right|^{\gamma} \leq \Phi\left(h^{\epsilon}\right) \quad \text { for } \gamma \geq 2
$$

we can assume that the initial height $h_{0}^{\epsilon}$ converges to a nonconstant function $h_{0}(x)$ depending on the space variable, so it is reasonable to expect that, as $\epsilon \rightarrow 0, h^{\epsilon} \rightarrow h_{0}$, and (1.1) yields the limit $\operatorname{div}\left(h_{0} u\right)=0$. The corresponding rotating lake equations are

$$
\begin{aligned}
& \operatorname{div}\left(h_{0} u\right)=0, \\
& \partial_{t}\left(h_{0} u\right)+\operatorname{div}\left(h_{0} u \otimes u\right)+h_{0} u^{\perp}+h_{0} \nabla \pi=0, \\
& \left.u\right|_{t=0}=u_{0}(x),\left.\quad h_{0} u \cdot n\right|_{\partial \Omega}=0, \quad \operatorname{div}\left(h_{0} u_{0}\right)=0 .
\end{aligned}
$$

Thus, roughly speaking, it is also reasonable to expect from the mathematical point of view that the smooth solution to (1.1)-(1.3) converges in suitable functional spaces to the smooth solution of (1.8)-(1.10) as $\epsilon \rightarrow 0$, and the hydrostatic pressure $\pi$ in (1.9) is the 'limit' of

$$
\frac{\left(h^{\epsilon}\right)^{\gamma-1}-h_{0}^{\gamma-1}}{\epsilon^{2}}
$$

in (1.2). This paper is devoted to the rigorous justification of the convergence of the above low Froude number limit for smooth solution of the inviscid rotating Euler and shallow water equations in a bounded two-dimensional domain $\Omega$. We remark that the existence and uniqueness of the classical solution of the lake equations (1.8)-(1.10) have been proved in [1].

Specifically, when $\gamma=2$, equations (1.1)-(1.3) comprise the rotating and inviscid shallow water system which models large scale geophysical motions in a thin layer of fluid under the influence of the Coriolis rotational forcing and is commonly used in oceanography and atmospheric physics [2-5]. For the singular limit problems of the shallow water model, we refer to $[6,7]$.

In [8], Cheng proved the singular limits and convergence rates of compressible Euler and rotating shallow water equations (1.1)-(1.3) with $h_{0}=1$ toward their incompressible counterparts. Recently, using the modulated energy method and by introducing a correction term which can serve as the acoustic part (density fluctuation) of the modulated energy, $\mathrm{Wu}$ [9] justified rigorously the convergence of the classical solution of the rotating shallow water model (1.1)-(1.3) to the classical solution of the rotating lake equations (or anelastic 
system) (1.8)-(1.10) when the Froude number tends to zero. However, no convergence rate was given in [9]. In this paper, our purpose is to refine the discussions in [9] and obtain the rate of convergence without introducing the correction term. Furthermore, we also prove the convergence of $\sqrt{h^{\epsilon}} u^{\epsilon}$ to $\sqrt{h_{0}} u$ with $\left\|\sqrt{h^{\epsilon}} u^{\epsilon}-\sqrt{h_{0}} u\right\|_{L^{\infty}\left([0, T] ; L^{2}(\Omega)\right)}^{2} \leq C \epsilon^{\frac{2}{\gamma}}$, which is not contained in [9].

In the following, we describe our main result. In order to simplify the presentation, we suppose that

$A_{1}: h_{0}^{\epsilon} \geq c>0,\left(h_{0}^{\epsilon}, h_{0}^{\epsilon} u_{0}^{\epsilon}\right) \in H^{3}(\Omega) \times\left(H^{3}(\Omega)\right)^{2}$ (ensuring the local-in-time existence of smooth solutions of system (1.1)-(1.2) and that $\left(h_{0}^{\epsilon}, h u_{0}^{\epsilon}\right)$ satisfy some appropriate compatibility conditions on $\partial \Omega$ );

$A_{2}: h_{0} \geq c>0, u_{0} \in\left(H^{3}(\Omega)\right)^{2}, \operatorname{div}\left(h_{0} u_{0}\right)=0$ (ensuring the local-in-time existence of smooth solutions of the rotating lake equations (1.8)-(1.9) and $\left(h_{0}, u_{0}\right)$ satisfy some appropriate compatibility conditions on $\partial \Omega$ ).

For a fixed $\epsilon>0$, the local existence and uniqueness of the classical solution of system (1.1)-(1.3) can be obtained under the assumptions $A_{1}$ and $A_{2}$.

Proposition 1.1 (Local existence of smooth solutions. (See [10])) Let $s \geq 3$ be an integer. Under the assumptions $A_{1}$ and $A_{2}$, there exist $T_{\epsilon}>0$ and a unique smooth solution $\left(h^{\epsilon}, u^{\epsilon}\right)$ to the system (1.1)-(1.3) defined in the time interval $\left[0, T_{\epsilon}\right]$, with

$$
\left(h^{\epsilon}, u^{\epsilon}\right) \in C^{1}\left(\left[0, T_{\epsilon}\right] ; H^{s}(\Omega)\right) \cap C\left(\left[0, T_{\epsilon}\right] ; H^{s+1}(\Omega)\right) .
$$

Now the main result of this paper reads as follows.

Theorem 1.2 Let $\left(h^{\epsilon}, u^{\epsilon}\right) \in H^{3}(\Omega) \times\left(H^{3}(\Omega)\right)^{2}$ be the smooth solution of system (1.1)-(1.3) established in Proposition 1.1 and let $u$ be a classical solution of the rotating lake equations (1.8)-(1.9) with the initial data $u_{0}$ which satisfies assumptions $A_{2}$ in $L^{\infty}\left(\left[0, T_{*}\right] ; H^{s}(\Omega)\right)$ and $T_{*}$ is the existence time of (1.8)-(1.9). Furthermore, we assume that the initial condition

$$
\frac{1}{\epsilon^{2}} \int_{\Omega} \Phi\left(h_{0}^{\epsilon}\right) d x+\int_{\Omega}\left|\sqrt{h_{0}^{\epsilon}} u_{0}^{\epsilon}-\sqrt{h_{0}} u_{0}\right|^{2} d x \leq C \epsilon^{\frac{2}{\gamma}}
$$

holds for some positive constant $C>0$. Then there exists a constant $C>0$, independent of $\epsilon$, such that as $\epsilon \rightarrow 0$, we have $T_{\epsilon} \geq T_{*}$ and the following estimate:

$$
\begin{aligned}
& \frac{1}{\epsilon^{2}}\left\|h^{\epsilon}-h_{0}\right\|_{L^{\infty}\left([0, T] ; L^{\gamma}(\Omega)\right)}^{\gamma} \leq C \epsilon^{\frac{2}{\gamma}}, \\
& \left\|\sqrt{h^{\epsilon}} u^{\epsilon}-\sqrt{h_{0}} u\right\|_{L^{\infty}\left([0, T] ; L^{2}(\Omega)\right)}^{2} \leq C \epsilon^{\frac{2}{\gamma}}, \\
& \left\|h^{\epsilon} u^{\epsilon}-h_{0} u\right\|_{L^{\infty}\left([0, T] ; L^{\left.\frac{2 \gamma}{\gamma+1}(\Omega)\right)}\right.}^{2} \leq C \epsilon^{\frac{2}{\gamma}}
\end{aligned}
$$

for all $T \in\left(0, T_{*}\right]$.

The proof of Theorem 1.2 is based on the modulated energy function. We will follow the same line as in [9]. However, to obtain the convergence rate, more delicate analyses on the energy functions are required. The next section is devoted to the rigorous proof of the main theorem. 


\section{Proof of the theorem}

We define $T^{\epsilon}=\min \left(T_{*}, T_{\epsilon}\right)>0$ so that the exact solution and the approximate solution are both defined in the time interval $\left[0, T^{\epsilon}\right]$.

From the energy (1.4) and the energy equality (1.6), we have

$$
\begin{aligned}
& \int_{\Omega}\left\{\frac{1}{2} h^{\epsilon}\left|u^{\epsilon}\right|^{2}+\frac{1}{\epsilon^{2}} \Phi\left(h^{\epsilon}\right)\right\} d x \\
& =\int_{\Omega}\left\{\frac{1}{2} h_{0}^{\epsilon}\left|u_{0}^{\epsilon}\right|^{2}+\frac{1}{\epsilon^{2}} \Phi\left(h_{0}^{\epsilon}\right)\right\} d x \leq C .
\end{aligned}
$$

Therefore, from (1.7), we have the following properties:

$$
\begin{aligned}
& \sqrt{h^{\epsilon}} u^{\epsilon} \quad \text { is bounded in } L^{\infty}\left(\left[0, T^{\epsilon}\right] ; L^{2}(\Omega)\right), \\
& \frac{1}{\epsilon^{2}}\left|h^{\epsilon}-h_{0}\right|^{\gamma} \quad \text { is bounded in } L^{\infty}\left(\left[0, T^{\epsilon}\right] ; L^{1}(\Omega)\right) .
\end{aligned}
$$

Thus from (2.3), we have

$$
\left\|h^{\epsilon}-h_{0}\right\|_{L^{\infty}\left(\left[0, T^{\epsilon}\right] ; L^{\gamma}(\Omega)\right)} \leq C \epsilon^{\frac{2}{\gamma}} .
$$

In fact, we will find that $\left\|h^{\epsilon}-h_{0}\right\|_{L^{\infty}\left(\left[0, T^{\epsilon}\right] ; L^{\gamma}(\Omega)\right)} \rightarrow 0$ has a faster rate of convergence.

Now we define the modulated energy functional $\mathcal{H}^{\epsilon}(t)$ as follows:

$$
\mathcal{H}^{\epsilon}(t)=\int_{\Omega}\left\{\frac{1}{2} h^{\epsilon}\left|u^{\epsilon}-u\right|^{2}+\frac{1}{\epsilon^{2}} \Phi\left(h^{\epsilon}\right)\right\} d x,
$$

where $u$ is the classical solution of the rotating lake equations (1.8)-(1.10). To derive the integration inequality for $\mathcal{H}^{\epsilon}(t)$, we use $u$ as a test function in equation (1.2) to yield the following equality:

$$
\begin{aligned}
-\int_{\Omega} h^{\epsilon} u^{\epsilon} \cdot u d x= & -\int_{\Omega}\left(h^{\epsilon} u^{\epsilon} \cdot u\right)(t=0) d x-\int_{0}^{t} \int_{\Omega} h^{\epsilon} u^{\epsilon} \cdot \partial_{s} u d x d s \\
& -\int_{0}^{t} \int_{\Omega} h^{\epsilon} u^{\epsilon} \otimes u^{\epsilon}: \nabla u d x d s+\int_{0}^{t} \int_{\Omega} h^{\epsilon}\left(u^{\epsilon}\right)^{\perp} \cdot u d x d s \\
& +\frac{1}{\epsilon^{2}} \int_{0}^{t} \int_{\Omega} h^{\epsilon} u \cdot \nabla\left(\left(h^{\epsilon}\right)^{\gamma-1}-h_{0}^{\gamma-1}\right) d x d s .
\end{aligned}
$$

From (1.8)-(1.9), we find the energy identity of the rotating lake equations:

$$
\frac{1}{2} \frac{d}{d t} \int_{\Omega} h_{0}|u|^{2} d x=0
$$

which implies that

$$
\int_{\Omega} h_{0}|u|^{2} d x=\int_{\Omega} h_{0}\left|u_{0}\right|^{2} d x
$$


Using (2.6)-(2.7) and the energy inequality (1.4), by integration by parts, we can calculate $\mathcal{H}^{\epsilon}(t)$ as follows:

$$
\begin{aligned}
\mathcal{H}^{\epsilon}(t)= & E^{\epsilon}(t)-\int_{\Omega} h^{\epsilon} u^{\epsilon} \cdot u d x+\frac{1}{2} \int_{\Omega} h^{\epsilon}|u|^{2} d x \\
= & \mathcal{H}^{\epsilon}(0)-\frac{1}{2} \int_{\Omega} h_{0}^{\epsilon}\left|u_{0}\right|^{2} d x+\frac{1}{2} \int_{\Omega} h^{\epsilon}|u|^{2} d x \\
& -\int_{0}^{t} \int_{\Omega} h^{\epsilon} u^{\epsilon} \otimes u^{\epsilon}: \nabla u d x d s+\int_{0}^{t} \int_{\Omega} h^{\epsilon}\left(u^{\epsilon}\right)^{\perp} \cdot u d x d s \\
& +\frac{1}{\epsilon^{2}} \int_{0}^{t} \int_{\Omega} h^{\epsilon} u \cdot \nabla\left(\left(h^{\epsilon}\right)^{\gamma-1}-h_{0}^{\gamma-1}\right) d x d s-\int_{0}^{t} \int_{\Omega} h^{\epsilon} u^{\epsilon} \cdot \partial_{s} u d x d s \\
= & \mathcal{H}^{\epsilon}(0)-\frac{1}{2} \int_{\Omega} h_{0}^{\epsilon}\left|u_{0}\right|^{2} d x+\frac{1}{2} \int_{\Omega} h^{\epsilon}|u|^{2} d x \\
& -\int_{0}^{t} \int_{\Omega} h^{\epsilon} u^{\epsilon} \cdot \partial_{s} u d x d s+\sum_{k=1}^{3} \mathcal{I}_{k},
\end{aligned}
$$

where

$$
\begin{aligned}
& \mathcal{I}_{1}=-\int_{0}^{t} \int_{\Omega} h^{\epsilon} u^{\epsilon} \otimes u^{\epsilon}: \nabla u d x d s, \\
& \mathcal{I}_{2}=\int_{0}^{t} \int_{\Omega} h^{\epsilon}\left(u^{\epsilon}\right)^{\perp} \cdot u d x d s, \\
& \mathcal{I}_{3}=\frac{1}{\epsilon^{2}} \int_{0}^{t} \int_{\Omega} h^{\epsilon} u \cdot \nabla\left(\left(h^{\epsilon}\right)^{\gamma-1}-h_{0}^{\gamma-1}\right) d x d s .
\end{aligned}
$$

It is noted that the first two terms on the right hand side of the above inequality is canceled in terms of (2.9).

Now, we begin to treat the integrals $\mathcal{I}_{k}(k=1,2,3,4)$ term by term. To deal with the kinetic part $\mathcal{I}_{1}$, we rewrite it as

$$
\begin{aligned}
\mathcal{I}_{1}= & -\int_{0}^{t} \int_{\Omega} h^{\epsilon}\left(u^{\epsilon}-u\right) \otimes\left(u^{\epsilon}-u\right): \nabla u d x d s \\
& -\int_{0}^{t} \int_{\Omega}\left(h^{\epsilon} u \otimes u^{\epsilon}\right): \nabla u d x d s \\
& +\int_{0}^{t} \int_{\Omega}\left(h^{\epsilon} u \otimes u\right): \nabla u d x d s-\int_{0}^{t} \int_{\Omega}\left(h^{\epsilon} u^{\epsilon} \otimes u\right): \nabla u d x d s \\
\leq & C \int_{0}^{t} \mathcal{H}^{\epsilon}(s) d s+\mathcal{I}_{11}+\mathcal{I}_{12},
\end{aligned}
$$

where

$$
\begin{aligned}
& \mathcal{I}_{11}=-\int_{0}^{t} \int_{\Omega}\left(h^{\epsilon} u \otimes u^{\epsilon}\right): \nabla u d x d s \\
& \mathcal{I}_{12}=\int_{0}^{t} \int_{\Omega}\left(h^{\epsilon} u \otimes u\right): \nabla u d x d s-\int_{0}^{t} \int_{\Omega}\left(h^{\epsilon} u^{\epsilon} \otimes u\right): \nabla u d x d s .
\end{aligned}
$$


Using integration by parts and the boundary conditions of $h^{\epsilon} u^{\epsilon}$ and $u$, we have

$$
\begin{aligned}
\mathcal{I}_{11} & =\frac{1}{2} \int_{0}^{t} \int_{\Omega}|u|^{2} \operatorname{div}\left(h^{\epsilon} u^{\epsilon}\right) d x d s \\
& =-\frac{1}{2} \int_{0}^{t} \int_{\Omega}|u|^{2} \partial_{s} h^{\epsilon} d x d s \\
& =-\frac{1}{2} \int_{0}^{t}\left(\frac{d}{d s} \int_{\Omega}|u|^{2} h^{\epsilon} d x\right) d s+\int_{0}^{t} \int_{\Omega} h^{\epsilon} u \cdot \partial_{s} u d x d s d x d s \\
& =\frac{1}{2} \int_{\Omega} h_{0}^{\epsilon}\left|u_{0}\right|^{2} d x-\frac{1}{2} \int_{\Omega} h^{\epsilon}|u|^{2} d x+\int_{0}^{t} \int_{\Omega} h^{\epsilon} u \cdot \partial_{s} u d x d s .
\end{aligned}
$$

Furthermore, we have

$$
\begin{aligned}
\mathcal{I}_{12} & =\int_{0}^{t} \int_{\Omega} h^{\epsilon} u_{i} u_{j} \partial_{j} u_{i} d x d s-\int_{0}^{t} \int_{\Omega} h^{\epsilon} u_{i}^{\epsilon} u_{j} \partial_{j} u_{i} d x d s \\
& =\int_{0}^{t} \int_{\Omega}\left(h^{\epsilon} u-h^{\epsilon} u^{\epsilon}\right) \cdot((u \cdot \nabla) u) d x d s .
\end{aligned}
$$

Therefore, we obtain

$$
\begin{aligned}
\mathcal{I}_{1} \leq & C \int_{0}^{t} \mathcal{H}^{\epsilon}(s) d s+\frac{1}{2} \int_{\Omega} h_{0}^{\epsilon}\left|u_{0}\right|^{2} d x-\frac{1}{2} \int_{\Omega} h^{\epsilon}|u|^{2} d x \\
& +\int_{0}^{t} \int_{\Omega} h^{\epsilon} u \cdot \partial_{s} u d x d s \\
& +\int_{0}^{t} \int_{\Omega}\left(h^{\epsilon} u-h^{\epsilon} u^{\epsilon}\right) \cdot((u \cdot \nabla) u) d x d s .
\end{aligned}
$$

Using the anti-symmetric property $\left(u^{\epsilon}\right)^{\perp} \cdot u=-u^{\epsilon} \cdot u^{\perp}$ and the orthogonal property $u \cdot u^{\perp}=0$, we obtain

$$
\mathcal{I}_{2}=\int_{0}^{t} \int_{\Omega}\left(h^{\epsilon} u-h^{\epsilon} u^{\epsilon}\right) \cdot u^{\perp} d x d s .
$$

Finally, we deal with the potential part $\mathcal{I}_{3}$. In view of (1.8) and the boundary condition of $u$, we have

$$
\begin{aligned}
& u \cdot \nabla h_{0}=-h_{0} \operatorname{div} u, \\
& \int_{0}^{t} \int_{\Omega} h_{0}^{\gamma} \operatorname{div} u d x d s=-\frac{\gamma}{\gamma-1} \int_{0}^{t} \int_{\Omega} h_{0} u \cdot \nabla h_{0}^{\gamma-1} d x d s=0 .
\end{aligned}
$$

Thus, we obtain

$$
\begin{aligned}
\mathcal{I}_{3} & =\frac{1}{\epsilon^{2}} \int_{0}^{t} \int_{\Omega} h^{\epsilon} u \cdot \nabla\left(h^{\epsilon}\right)^{\gamma-1} d x d s-\frac{1}{\epsilon^{2}} \int_{0}^{t} \int_{\Omega} h^{\epsilon} u \cdot \nabla h_{0}^{\gamma-1} d x d s \\
& =\frac{\gamma-1}{\gamma \epsilon^{2}} \int_{0}^{t} \int_{\Omega} u \cdot \nabla\left(h^{\epsilon}\right)^{\gamma} d x d s-\frac{\gamma-1}{\epsilon^{2}} \int_{0}^{t} \int_{\Omega} h^{\epsilon} h_{0}^{\gamma-2} u \cdot \nabla h_{0} d x d s \\
& =-\frac{\gamma-1}{\gamma \epsilon^{2}} \int_{0}^{t} \int_{\Omega}\left(h^{\epsilon}\right)^{\gamma} \operatorname{div} u d x d s+\frac{\gamma-1}{\epsilon^{2}} \int_{0}^{t} \int_{\Omega} h^{\epsilon} h_{0}^{\gamma-1} \operatorname{div} u d x d s
\end{aligned}
$$




$$
\begin{aligned}
& =-\frac{\gamma-1}{\gamma \epsilon^{2}} \int_{0}^{t} \int_{\Omega}\left[\left(h^{\epsilon}\right)^{\gamma}-\gamma h^{\epsilon} h_{0}^{\gamma-1}+(\gamma-1) h_{0}^{\gamma}\right] \operatorname{div} u d x d s \\
& \leq C \int_{0}^{t} \mathcal{H}^{\epsilon}(s) d s .
\end{aligned}
$$

Inserting the estimates (2.9)-(2.11) into (2.8), we have, from (1.9),

$$
\begin{aligned}
\mathcal{H}^{\epsilon}(t)= & \mathcal{H}^{\epsilon}(0)+C \int_{0}^{t} \mathcal{H}^{\epsilon}(s) d s \\
& +\int_{0}^{t} \int_{\Omega}\left(h^{\epsilon} u-h^{\epsilon} u^{\epsilon}\right)\left[\partial_{s} u+(u \cdot \nabla u) u+u^{\perp}\right] d x d s \\
= & \mathcal{H}^{\epsilon}(0)+C \int_{0}^{t} \mathcal{H}^{\epsilon}(s) d s+\int_{0}^{t} \int_{\Omega}\left(h^{\epsilon} u^{\epsilon}-h^{\epsilon} u\right) \cdot \nabla \pi d x d s .
\end{aligned}
$$

From the continuity equation (1.1), the initial conditions (1.11), the estimate (2.13), and the Hölder inequality, we get, by integration by parts,

$$
\begin{aligned}
\int_{0}^{t} \int_{\Omega} h^{\epsilon} u^{\epsilon} \cdot \nabla \pi d x d s= & \int_{\Omega}\left(h^{\epsilon}-h_{0}\right) \pi d x-\int_{\Omega}\left(h_{0}^{\epsilon}-h_{0}\right) \pi_{0} d x \\
& -\int_{0}^{t} \int_{\Omega}\left(h^{\epsilon}-h_{0}\right) \partial_{s} \pi d x d s \\
\leq & \left\|h^{\epsilon}-h_{0}\right\|_{L^{\gamma}(\Omega)}\|\pi\|_{L^{\frac{\gamma}{\gamma-1}(\Omega)}}+\left\|h_{0}^{\epsilon}-h_{0}\right\|_{L^{\gamma}(\Omega)}\left\|\pi_{0}\right\|_{L^{\frac{\gamma}{\gamma-1}}(\Omega)} \\
& +\left\|h^{\epsilon}-h_{0}\right\|_{L^{\infty}\left(\left[0, T^{\epsilon}\right] L^{\gamma}(\Omega)\right)}\left\|\partial_{t} \pi\right\|_{L^{\infty}\left(\left[0, T^{\epsilon}\right] ; L^{\gamma-1}\right.} \frac{\gamma}{\gamma(\Omega))} \\
\leq & C \epsilon^{\frac{2}{\gamma}}
\end{aligned}
$$

and

$$
\begin{aligned}
-\int_{0}^{t} \int_{\Omega} h^{\epsilon} u \cdot \nabla \pi d x d s= & -\int_{0}^{t} \int_{\Omega}\left(h^{\epsilon}-h_{0}\right) u \cdot \nabla \pi d x d s \\
\leq & \left\|h^{\epsilon}-h_{0}\right\|_{L^{\infty}\left(\left[0, T^{\epsilon}\right] ; L^{\gamma}(\Omega)\right)}\|u\|_{L^{\infty}\left(\left[0, T^{\epsilon}\right] ; L^{\gamma-1}\right.} \frac{2 \gamma}{\gamma(\Omega))} \\
& \times\|\nabla \pi\|_{L^{\infty}\left(\left[0, T^{\epsilon}\right] ; L^{\frac{2 \gamma}{\gamma-1}}(\Omega)\right)} \\
\leq & C \epsilon^{\frac{2}{\gamma}}
\end{aligned}
$$

Combining (2.12) with (2.13)-(2.14), one gets

$$
\mathcal{H}^{\epsilon}(t) \leq \mathcal{H}^{\epsilon}(0)+C \int_{0}^{t} \mathcal{H}^{\epsilon}(s) d s+C \epsilon^{\frac{2}{\gamma}}, \quad \forall t \in\left(0, T^{\epsilon}\right]
$$

Using the initial conditions (1.11), we have $\mathcal{H}^{\epsilon}(0) \leq C \epsilon^{\frac{2}{\gamma}}$ since

$$
\begin{aligned}
\int_{\Omega} h_{0}^{\epsilon}\left|u_{0}^{\epsilon}-u_{0}\right|^{2} d x & \leq 2 \int_{\Omega}\left|\sqrt{h_{0}^{\epsilon}} u_{0}^{\epsilon}-\sqrt{h_{0}} u_{0}\right|^{2} d x+2 \int_{\Omega}\left|\left(\sqrt{h_{0}}-\sqrt{h_{0}^{\epsilon}}\right) u_{0}\right|^{2} d x \\
& \leq 2\left\|\sqrt{h_{0}^{\epsilon}} u_{0}^{\epsilon}-\sqrt{h_{0}} u_{0}\right\|_{L^{2}(\Omega)}^{2}+2\left\|u_{0}\right\|_{L^{\infty}(\Omega)}^{2}\left\|\sqrt{h_{0}^{\epsilon}}-\sqrt{h_{0}}\right\|_{L^{2}(\Omega)}^{2}
\end{aligned}
$$




$$
\begin{aligned}
& \leq 2\left\|\sqrt{h_{0}^{\epsilon}} u_{0}^{\epsilon}-\sqrt{h_{0}} u_{0}\right\|_{L^{2}(\Omega)}^{2}+C\left\|h_{0}^{\epsilon}-h_{0}\right\|_{L^{\gamma}(\Omega)}^{2} \\
& \leq C \epsilon^{\frac{2}{\gamma}}
\end{aligned}
$$

Here, we also have used assumption $\left(A_{1}\right)$ and the following elementary inequality:

$$
\left|\sqrt{x_{1}}-\sqrt{x_{2}}\right|^{2} \leq \frac{1}{c}\left|x_{1}-x_{2}\right|^{2}, \quad \forall x_{1}, x_{2} \geq c>0,
$$

the Hölder inequality and the finite measure of $\Omega$. Then, with the help of the Gronwall inequality, we get

$$
\mathcal{H}^{\epsilon}(t) \leq C \epsilon^{\frac{2}{\gamma}}, \quad \forall t \in\left(0, T^{\epsilon}\right]
$$

Using the Hölder inequality, we have

$$
\begin{aligned}
\left\|\sqrt{h^{\epsilon}} u^{\epsilon}-\sqrt{h_{0}} u\right\|_{L^{2}(\Omega)}^{2} & \leq 2\left\|\sqrt{h^{\epsilon}}\left(u^{\epsilon}-u\right)\right\|_{L^{2}(\Omega)}^{2}+2\left\|\left(\sqrt{h^{\epsilon}}-\sqrt{h_{0}}\right) u\right\|_{L^{2}(\Omega)}^{2} \\
& \leq C \epsilon^{\frac{2}{\gamma}}+C\left\|\sqrt{h^{\epsilon}}-\sqrt{h_{0}}\right\|_{L^{2}(\Omega)}^{2}\|u\|_{L^{\infty}(\Omega)}^{2} \\
& \leq C \epsilon^{\frac{2}{\gamma}}
\end{aligned}
$$

for any $t \in\left(0, T^{\epsilon}\right]$. In view of (1.12) and using the Hölder inequality, we have

$$
\begin{aligned}
\left\|h^{\epsilon} u^{\epsilon}-h_{0} u\right\|_{L^{\frac{2 \gamma}{\gamma+1}}(\Omega)}^{2} \leq & 2\left\|h^{\epsilon}\left(u^{\epsilon}-u\right)\right\|_{L^{\frac{2 \gamma}{\gamma+1}(\Omega)}}^{2}+2\left\|\left(h^{\epsilon}-h_{0}\right) u\right\|_{L^{\frac{2 \gamma}{\gamma+1}}(\Omega)}^{2} \\
\leq & 2\left\|\sqrt{h^{\epsilon}}\right\|_{L^{2 \gamma}(\Omega)}^{2}\left\|\sqrt{h^{\epsilon}}\left(u^{\epsilon}-u\right)\right\|_{L^{2}(\Omega)}^{2} \\
& +2\left\|\sqrt{h^{\epsilon}}-\sqrt{h_{0}}\right\|_{L^{\gamma}(\Omega)}^{2}\|u\|_{L^{\frac{\gamma}{\gamma-1}}(\Omega)}^{2} \\
\leq & C \epsilon^{\frac{2}{\gamma}}
\end{aligned}
$$

for any $t \in\left(0, T^{\epsilon}\right]$. By a standard argument on the time extension of smooth solutions, we obtain $T^{\epsilon} \geq T_{*}$, i.e. $T^{\epsilon}=T_{*}$. With the aid of (1.7) and (2.16)-(2.18), we conclude that the proof of Theorem 1.2 is finished.

\section{Competing interests}

The authors declare that they have no competing interests.

\section{Authors' contributions}

All authors carried out the proof. All authors read and approved the final manuscript.

\section{Author details}

'Institute of Water Resources and Hydro-electric Engineering, Xi'an University of Technology, Xi'an, Shanxi Province 710048, P.R. China. ${ }^{2}$ College of Mathematics and Information Science, North China University of Water Resources and Electric Power, Zhengzhou, Henan Province 450045, P.R. China.

\section{Acknowledgements}

J. Yang's research was partially supported by the Joint Funds of the National Natural Science Foundation of China (Grant No. U1204103) 


\section{References}

1. Levermore, CD, Oliver, M, Titi, ES: Global well-posedness for models of shallow water in a basin with a varying bottom. Indiana Univ. Math. J. 45, 479-510 (1996)

2. Ghil, M, Childress, S: Topics in Geophysical Fluid Dynamics: Atmospheric Dynamics, Dynamo Theory, and Climate Dynamics. Applied Mathematical Sciences, vol. 60. Springer, New York (1987)

3. Majda, A: Introduction to PDEs and Waves for the Atmosphere and Ocean, vol. 9. Am. Math. Soc., Providence (2003)

4. Oliver, M: Variational asymptotics for rotating shallow water near geostrophy: a transformational approach. J. Fluid Mech. 551, 197-234 (2006)

5. Pedlosky, J: Geophysical Fluid Dynamics. Springer, Berlin (1992)

6. Bernardi, C, Pironneau, O: On the shallow water equations at low Reynolds number. Commun. Partial Differ. Equ. 16 59-104 (1991)

7. Bresch, D, Desjardins, B: Existence of global weak solutions for a $2 \mathrm{D}$ viscous shallow water equations and convergence to the quasi-geostrophic model. Commun. Math. Phys. 238, 211-223 (2003)

8. Cheng, B: Singular limits and convergence rates of compressible Euler and rotating shallow water equations. SIAM J. Math. Anal. 44, 1050-1076 (2012)

9. Wu, KC: Low Froude number limit of the rotating shallow water and Euler equations. Proc. Am. Math. Soc. 142, 939-947 (2014)

10. Beirão da Veiga, H: Data dependence in the mathematical theory of compressible inviscid fluids. Arch. Ration. Mech. Anal. 119, 109-127 (1992)

\section{Submit your manuscript to a SpringerOpen ${ }^{\circ}$ journal and benefit from:}

- Convenient online submission

Rigorous peer review

- Immediate publication on acceptance

- Open access: articles freely available online

High visibility within the field

- Retaining the copyright to your article 